\title{
Revisão da literatura
}

\section{Os efeitos do estresse na função do eixo hipotalâmico-pituitário-adrenal em indivíduos com esquizofrenia}

\author{
The effects of stress on hypothalamic-pituitary-adrenal (HPA) axis function in subjects with schizophrenia \\ Francesca L. Guest'1, Daniel Martins-de-Souza 23,3 , Hassan Rahmoune2, Sabine Bahn²,4, Paul C. Guest² \\ - Faculdade de Medicina e Odontologia, Universidade de Bristol, Bristol, Reino Unido. \\ 2 Departamento de Engenharia Química e Biotecnologia, Universidade de Cambridge, Cambridge, Reino Unido. \\ 3 Instituto Max Planck de Psiquiatria e Universidade Ludwig Maximilians de Munique (LMU), Alemanha; Laboratório de Neurociências (LIM-27), Instituto de Psiquiatria, Faculdade de Medicina da \\ Universidade de São Paulo (IPq-FMUSP), Brasil. \\ ${ }_{4}^{4}$ Departamento de Neurociências, Centro Médico Erasmus, Holanda.
}

Recebido: 23/9/2012 - Aceito: 7/11/2012

\begin{abstract}
Resumo
Nas últimas décadas, têm surgido evidências sugerindo que a patogênese de desordens psiquiátricas, tais como a esquizofrenia, pode envolver perturbações no eixo hipotalâmico-pituitário-adrenal (HPA). Variações na manifestação desses efeitos poderiam estar relacionadas a diferenças em sintomas clínicos entre os indivíduos afetados, assim como a diferenças na resposta ao tratamento. Tais efeitos podem também ser originados de complexas interações entre genes e fatores ambientais. Aqui, revisamos os efeitos do estresse maternal em anormalidades na regulação do eixo HPA e desenvolvimento de desordens psiquiátricas, incluindo a esquizofrenia. Estudos nessa área podem gerar o aumento do nosso entendimento da natureza multidimensional da esquizofrenia. Posterior pesquisa nesse campo poderia, em última instância, levar ao desenvolvimento de melhores diagnósticos e novas abordagens terapêuticas para essa debilitante condição psiquiátrica.
\end{abstract}

Guest FL, et al. / Rev Psiq Clín. 2013;40(1):20-7

Palavras-chave: Desordens psiquiátricas, esquizofrenia, disfunção do eixo HPA, diagnóstico, biomarcadores.

\begin{abstract}
Over the last few decades, evidence has been emerging that the pathogenesis of psychiatric disorders such as schizophrenia can involve perturbations of the hypothalamic-pituitary-adrenal (HPA) axis. Variations in the manifestation of these effects could be related to the differences in clinical symptoms between affected individuals as well as to differences in treatment response. Such effects can also arise from the complex interaction between genes and environmental factors. Here, we review the effects of maternal stress on abnormalities in HPA axis regulation and the development of psychiatric disorders including schizophrenia. Studies in this area may prove critical for increasing our understanding of the multi-dimensional nature of schizophrenia. Further research in this area could ultimately lead to the development of improved diagnostics and novel therapeutic approaches for treating this debilitating psychiatric condition.
\end{abstract}

Guest FL, et al. / Rev Psiq Clín. 2013;40(1):20-7

Keywords: Psychiatric disorders, schizophrenia, HPA axis dysfunction, diagnosis, biomarkers.

\section{Introdução}

Durante um evento estressante, substâncias químicas como adrenalina e glicocorticoides são lançadas na corrente sanguínea, levando a uma cadeia de reações no corpo. O efeito dessas substâncias de situações de estresse, quando experimentadas durante a gravidez, tem sido investigado. David Barker propôs a "hipótese da programação fetal" - também conhecida simplesmente como a hipótese de Barker - no seu artigo de 1989, que detalha a importância do ambiente intrauterino no desenvolvimento de órgãos e tecidos fetais ${ }^{1}$. Essa hipótese sugere que um desvio do ambiente intrauterino ideal pode ter efeitos permanentes na função do órgão ou estrutura ${ }^{1,2}$. Extrapolação desse conceito ao cérebro sugere que durante o primeiro e o segundo trimestre de gravidez o ambiente intrauterino terá efeitos diretos na estrutura do cérebro do feto, os quais podem perdurar por toda a vida adulta.

Correlações entre o estresse maternal pré-natal e anormalidades comportamentais e psicológicas produzidas na prole animal têm sido amplamente documentadas. Foi proposto que hormônios lançados na corrente sanguínea, em resposta a um estímulo estressante percebido pela mãe, têm efeito direto no desenvolvimento do cérebro e outros órgãos do feto. A correta regulação da liberação hormonal na gravidez é crítica para o correto desenvolvimento fetal, e qualquer desvio da concentração normal desses hormônios pode produzir alterações microscópicas e macroscópicas no cérebro, particularmente na conectividade sináptica dentro de regiões cerebrais distintas e entre elas ${ }^{3}$. Entretanto, não é um estressor agudo, mas o estresse crônico que predispõe a desordens psiquiátricas na prole. Acredita-se que desenvolvimento de problemas psicológicos e comportamentais, incluindo autismo, transtorno de déficit de atenção com hiperatividade (TDAH), desordem depressiva maior, transtorno bipolar e esquizofrenia, estejam ligados a tais perturbações no eixo hipotalâmico-pituitário-adrenal (HPA) e em outros órgãos do sistema neuroendócrino difuso.

Na pesquisa realizada por outros grupos, há clara evidência entre o estresse maternal e problemas comportamentais e psicológicos na prole, apesar de não se acreditar ser algo puramente hormonal. Também se especula que ligações genéticas possam existir em muitas dessas doenças e que estas possam ser, de alguma forma, hereditárias. Deveria ser considerado também que mães com problemas psicológicos ou que possuem problemas em administrar o estresse podem ser mais propensas a se tornarem estressadas no período da gravidez e, dessa forma, induzem uma condição similar em sua prole. É mais provável que esses problemas apresentem ambas as causas: genética e hormonal. A hipótese de duas causas ${ }^{4}$ sugere que uma predisposição genética existe no feto e, então, um ambiente hormonal anômalo no útero permitiria a materialização de uma anormalidade. Esta revisão objetiva resumir e correlacionar a literatura existente a respeito do estresse pré-natal no desenvolvimento do cérebro do feto e como isso pode afetar o comportamento, a psicologia e a saúde da ninhada 
mais tarde na vida. Como muito da literatura atual reporta o uso de modelos animais para testar essas hipóteses, especularemos em que extensão tais achados podem ser extrapolados para humanos. Também examinaremos os prováveis efeitos do estresse pré-natal no desenvolvimento de transtornos psiquiátricos em humanos e discutiremos os efeitos relatados daquele na função do eixo HPA e outros sistemas neuroendócrinos. Finalmente, questionaremos a importância de quando se dá o estímulo estressor em relação ao período gestacional materno, bem como o impacto dele na existência e severidade de problemas comportamentais na prole.

\section{Estresse: definições}

O livro Neuroendocrinologia, uma abordagem integrada descreve estresse como "qualquer evento, quer real ou percebido, que age promovendo um distúrbio do equilíbrio homeostático"s. Quando pensamos em estresse no nosso dia a dia, tendemos a imaginar o estresse emocional. Isso poderia significar os efeitos de prazos a cumprir, testes importantes, chefes exigentes e importantes decisões que podem ser incluídos como estímulos estressores ${ }^{6}$. Nem todos respondem ao estresse da mesma maneira, assim como não possuem o mesmo limiar para o estresse a ser suportado ${ }^{7,8}$. Algumas pessoas se sentem estressadas somente em situações extremas, já outras podem se mostrar extremamente estressadas por assuntos considerados de menor importância. O estresse pode ser produzido por medo do desconhecido, do imprevisto e das possíveis más consequências. Pode-se citar, por exemplo, o medo de que o ser amado sofra um acidente, muito embora não haja evidência que sugira a possibilidade de tal acontecimento. Os mais intensos estresses emocionais ocorrem em relações de proximidade, como entre parentes ou parceiros ${ }^{9}$. Outros tipos de estresse incluem aqueles de natureza mecânica ou física, tais como lesões por impacto. Embora isso possa levar ao estresse emocional em muitas circunstâncias, o estresse mecânico não é necessariamente relevante ao assunto desta revisão, já que esta é mais focada em estressores bioquímicos e emocionais. Definições simples de estresse bioquímico poderiam ser a adição de uma substância tóxica, a privação de uma substância necessária ao corpo ou qualquer outro desvio da normalidade. Exemplos incluem vida em alta altitude, dieta pobre em proteínas ou exposição a extremos de temperatura.

A extensão em que um estresse em particular afetará o indivíduo pode depender simplesmente da capacidade do indivíduo em lidar com esse estresse. Uma pessoa que se sente aprisionada e incapaz de suportar um evento estressante pode experimentar uma sensação de depressão ou sofrimento, enquanto uma pequena quantidade de estresse pode levar outra pessoa a trabalhar mais, não perder as esperanças ou promover um sentimento de bem-estar ${ }^{10}$. Um exemplo do último seria a finalização de um projeto antes do seu prazo final, sendo auxiliado pela pressão por fazê-lo. Isso tem sido descrito como "eustress"11. Algumas pessoas se descrevem como funcionários mais produtivos sob pressão e, dessa forma, pode ser que entre eles se encontrem aqueles que suportam os maiores níveis de estresse.

\section{A resposta ao estresse em humanos}

Em reação a um estímulo produzido por estresse, o corpo produz uma resposta por meio da ativação do eixo HPA. Esse é um mecanismo necessário, já que prepara o corpo para a bem conhecida reação de "luta ou fuga" perante uma situação potencialmente perigosa12. Entretanto, a reação ao estresse produzida não difere grandemente dependendo do agente estressor. Em humanos, as principais moléculas responsáveis pela resposta ao estresse são a adrenalina e glicocorticoides ${ }^{13}$. Existem três fases principais de uma resposta geral ao estresse, as quais são colocadas em ação quando o estressor é detectado. A primeira fase envolve a produção de moléculas catecolaminas adrenalina e noradrenalina. Esses são exemplos de hormônios monoaminas, que são derivados do aminoácido tirosina. A conversão de tirosina em di-hidroxifenilalanina (DOPA) acontece nas células cromafinas, encontradas na medula adrenal ${ }^{14}$. Noradrenalina e adrenalina são liberadas da medula adrenal quase imediatamente na corrente sanguínea via sistema nervoso autônomo e afetam diversas respostas no corpo. Por exemplo, a liberação de adrenalina e noradrenalina causa aumento na frequência de batimentos cardíacos, vasoconstrição, dilatação das pupilas (midríase) e abertura das passagens respiratórias. Em adição, a secreção de insulina é inibida e a glicogenólise é estimulada nos músculos e fígado, levando a uma maior concentração de glicose na corrente sanguínea ${ }^{15}$.

Secundariamente, após 20 minutos, o fator liberador de corticotrofina (CRF) é liberado do núcleo paraventricular (PVN) do hipotálamo no sistema portal e na eminência mediana do hipotálamo. Este, por sua vez, estimula a liberação do hormônio adrenocorticotrófico $(\mathrm{ACTH})$ da pituitária anterior diretamente na corrente sanguínea. O ACTH viaja pela circulação até as glândulas adrenais, culminando com a liberação de glicocorticoides como o cortisol do córtex da adrenal. No que se refere ao estresse pré-natal em humanos, o cortisol é o mais importante glicocorticoide. A elevação dos níveis de cortisol no sangue resulta na inibição da liberação de ACTH e CRF em um mecanismo de retroalimentação negativa ${ }^{5}$.

No terceiro estágio, glicocorticoides produzem respostas similares àquelas das catecolaminas, incluindo aumento da gliconeogênese e lipólise, levando a maiores volumes de glicerol e ácidos graxos na corrente sanguínea. Glicocorticoides também estimulam a mobilização e desaminação de proteínas do músculo esquelético ${ }^{5}$.

\section{Programação fetal em resposta ao estresse}

O estresse pré-natal crônico está ligado a mudanças morfológicas no cérebro fetal, mas os mecanismos responsáveis por esses efeitos não são bem conhecidos. Os eixos HPA maternal e fetal e a placenta têm sido estudados para determinar se podem servir como condutores para tais efeitos morfológicos ${ }^{16}$. Durante a gravidez o corpo da mãe passa por muitas mudanças físicas e químicas, incluindo a produção alterada de certos hormônios. Os níveis de cortisol são tipicamente encontrados mais altos em uma mãe grávida ${ }^{17}$. Tal elevação do cortisol é essencial para o crescimento do feto e estimula a produção de surfactante. Entretanto, excessiva elevação dos níveis de cortisol pode modificar permanentemente o crescimento do feto ${ }^{18}$. O estresse altera os níveis de muitos hormônios e outras moléculas encontradas no sangue, e isso pode acarretar modificações no feto. $\mathrm{O}$ estresse pré-natal afeta em particular a placenta, diminuindo a função desse órgão por meio de uma redução dos níveis de nutrientes e oxigênio que alcançam o feto. O mecanismo desse efeito resulta de níveis elevados de adrenalina no sangue, os quais induzem o aumento da resistência vascular e a redução do fluxo de sangue para a placenta ${ }^{16}$.

O feto possui uma barreira natural enzimática ao cortisol chamada $11 \beta$-hidroxiesteroide desidrogenase-2 (11 $\beta$-HSD2) ${ }^{16}$. Essa molécula é menos expressa em casos de estresse maternal, deixando o feto mais suscetível à exposição ao cortisol. A redução nos níveis de $11 \beta$-HSD2 também leva a um aumento na produção de outros hormônios tais como prostaglandinas, estrogênio e lactogênio placentário $^{16}$. Além disso, os níveis de $\mathrm{CRH}$ placentários estão sujeitos à regulação pelo eixo HPA maternal ${ }^{19}$. Estudos mostraram que aumentos das concentrações de CRH induzidos por estresse podem levar a mudanças macroscópicas e microscópicas do hipocampo da ninhada ${ }^{20-22}$

\section{Problemas comportamentais e psicológicos}

Um grande número de diferentes problemas psicológicos tem se mostrado ligado ao estresse antenatal. Um aumento na incidência de transtorno de déficit de atenção com hiperatividade (TDAH) foi encontrado em ninhadas de macacos rhesus quando as mães foram submetidas a barulhos altos durante a gravide $z^{23,24}$. Estudos mostraram que ninhadas de mães expostas a estresse prolongado apresentaram maiores respostas aos estressores, anormalidades em lidar com o estresse e comportamento social perturbado 25,26 . A incidência de TDAH também apresentou aumento nas ninhadas nas quais as mães experimentaram privação durante a gravidez, como mostrado em um estudo dinamarquês ${ }^{27}$. Nesse caso, privação inesperada resultou 
em $72 \%$ de aumento na probabilidade de ocorrência de TDAH na ninhada. Muitos outros pesquisadores reportaram maior prevalência de TDAH nas ninhadas de mães estressadas ${ }^{28,29}$.

Um grande número de estudos tem sugerido que há aumento na incidência de esquizofrenia de ninhadas de mães estressadas no período pré-natal. A invasão do exército alemão à Holanda, em maio de 1940, levou a um estresse de proporções nacionais. Um grupo consistindo de ninhadas de mães expostas a esse estresse durante o primeiro, o segundo e o terceiro trimestre de gravidez foi acompanhado e a prevalência de esquizofrenia foi observada ${ }^{30}$. Isso revelou uma maior incidência de esquizofrenia entre esses indivíduos, com o maior aumento visto naquelas mães expostas durante o primeiro trimestre ${ }^{31-34}$. Achados similares resultaram da Fome Chinesa de 1958-196135. Novamente, aqueles indivíduos concebidos no pico da escassez alimentar apresentaram maior risco de esquizofrenia e outras condições. Maior incidência de esquizofrenia na ninhada de mães estressadas também foi relatada por Huttenen et al. ${ }^{36}$, Kinney et al. ${ }^{37} \mathrm{e}$ outros.

Posterior evidência suportando a importância do ambiente intrauterino na predisposição de doenças psiquiátricas foi vista em modelos animais. Por exemplo, estudos envolvendo a privação de proteína no período pré-natal em ratos mostrou que isso pode levar a efeitos crônicos na ninhada. Esses efeitos incluem mudanças na morfologia hipocampal e na ligação de glutamato e dopamina a seus receptores ${ }^{38}$. Outros estudos mostram que a restrição proteica durante a gravidez pode ter efeito negativo no desenvolvimento cerebral fetal. Acredita-se que isso se deva a mudanças no metabolismo maternal de lipídeos, os quais são componentes essenciais de membranas celulares ${ }^{39}$. Interessantemente, as anormalidades comportamentais vistas nesses modelos com restrição proteica não se manifestaram até a idade adulta, incluindo efeitos nas atividades cérebro-reguladas, tais como diminuição da inibição pré-pulso da resposta de alarme e hiperlocomoção, as quais são tradicionalmente usadas como medidas de comportamento do tipo esquizofrênico ${ }^{40,41}$. Um pré-pulso é uma versão reduzida de um estímulo entregue a um indivíduo imediatamente antes de uma versão completa do estímulo (pulso) ser aplicada. O cérebro normalmente regulará negativamente sua resposta-alarme a um estímulo se esse for precedido por um pré-pulso. Entretanto, indivíduos com esquizofrenia ou doença de Alzheimer mostrarão uma resposta-alarme da mesma magnitude independentemente de um pré-pulso ${ }^{40-42}$.

Watson et al. ${ }^{43}$ reportaram incidência de depressão de $13,3 \%$ na prole de mães que foram expostas a um severo terremoto comparada a uma incidência de 5,5\% vistos em prole de mães não expostas. Depressão também tem sido ligada a anormalidades vistas no eixo HPA de ratas prenhes sofrendo de estresse pré-natal ${ }^{44}$. Depois de a Louisiana ter sido atingida por uma série de furacões e tempestades tropicais, um estudo examinando as diferenças entre a incidências de autismo na prole das mães foi realizado ${ }^{45}$. Ele mostrou uma incidência de 26,59/10.000 no grupo de alta exposição, comparado a $3,72 / 10.000$ no grupo de baixa exposição. Beversdorf et al. ${ }^{4}$ também estabeleceram uma ligação entre estresse pré-natal e autismo. Um grande número de outras notáveis anormalidades tem sido relacionado ao estresse pré-natal na mãe, incluindo questões cognitivas, comportamentais e emocionais ${ }^{47-51}$. Interessantemente, estudos em ratos mostram que ninhadas de mães que foram confinadas na última semana de gestação apresentam maior probabilidade de autoadministração de drogas, tais como cocaína e anfetaminas, do que daquelas de mães não confinadas ${ }^{52}$.

\section{Mudanças microscópicas e macroscópicas no cérebro}

Regiões específicas do cérebro têm mostrado ser afetadas pelo estresse pré-natal, tanto macroscópica quanto microscopicamente. Essas regiões incluem hipocampo ${ }^{53-57}$, amígdala ${ }^{58-60}$, corpo caloso ${ }^{61}$, córtex cerebral ${ }^{62,63}$, cerebelo ${ }^{64,65}$ e hipotálamo ${ }^{66,67}$. Já que mudanças morfológicas nas regiões cerebrais acima têm sido ligadas a certos problemas psicológicos e comportamentais, é possível que o estresse pré-natal afete o desenvolvimento neurológico dessas áreas. Isso, por sua vez, estaria relacionado a efeitos comportamentais, como aqueles vistos nas desordens psiquiátricas.

Mudanças no tamanho do corpo caloso e no número de tipos celulares específicos dentro dessa estrutura cerebral têm sido relacionadas a autismo ${ }^{68}, \mathrm{TDAH}^{69}$ e esquizofrenia ${ }^{70}$. Ligações do autismo, TDAH e esquizofrenia ao estresse pré-natal foram detalhadas anteriormente. Dessa forma, pode ser razoável extrapolar esses achados para sugerir que o estresse pré-natal afeta diretamente a estrutura do corpo caloso. Entretanto, nenhum estudo nessa área foi realizado em humanos.

O hipocampo é conhecido por estar envolvido na formação da memória e desempenha papel fundamental no aprendizado. Lemaire et al. ${ }^{71}$ reportaram que o estresse pré-natal tinha um efeito negativo na memória de ratos. Os neurônios granulares hipocampais continuam a ser criados durante a vida e são responsáveis pela formação de memórias. Entretanto, o número de tais neurônios granulares foi reduzido em ratos adultos cujas mães foram expostas a estresse durante a gravidez. Em adição, os autores mostraram que o estresse pré-natal reduziu a proliferação de células hipocampais e a taxa de sobrevivência das novas células, juntamente com a redução do número de neurônios diferenciados. De maneira interessante, todos esses efeitos deletérios poderiam ser contornados pela manipulação neonata.

Embora modelos animais tenham levado à compreensão parcial da conexão de alterações morfológicas no cérebro, estresse pré-natal e desordens comportamentais, não se pode assumir que os mesmos efeitos ocorram em humanos. Na verdade, isso não é provável que aconteça por causa das largas diferenças com relação ao tempo e à extensão com que ocorre o desenvolvimento do cérebro antes, durante e após o nascimento nas diferentes espécies animais.

\section{Tempo de ocorrência e gravidade}

Em ratos e primatas não humanos, o tempo em que o estressor se apresenta na gravidez se mostrou importante ${ }^{58-60}$. Um estudo usando ratos mostrou $64 \%$ de aumento na produção de corticosterona - um hormônio similar à aldosterona em humanos - após manipulação de ratas prenhes na última semana de gestação, colocando-as em gaiolas novas e não familiares a elas ${ }^{72}$. Apesar de alguma evidência ter sido apresentada no caso de modelos animais, o tempo de ocorrência do estressor e a severidade com que o feto humano é afetado são mais difíceis de determinar. Essa dificuldade se deve principalmente ao fato de que seria considerada antiética a exposição de mulheres grávidas a um estresse em diferentes pontos durante a gravidez. Entretanto, desastres naturais podem servir a esse propósito; tais eventos podem prover um grande grupo de pessoas afetadas por um estresse idêntico. Isso permite uma análise retrospectiva daquelas que estiveram grávidas durante o desastre natural e estudos subsequentes da prole. Dessa forma, pode-se concluir quando qualquer desses padrões vistos nos modelos animais pode ser extrapolado para humanos.

Um estudo chamado "Projeto Tempestade de Gelo" tentou determinar o tempo de ocorrência dos efeitos do estresse maternal na prole usando humanos. Nesse caso mais de 3 milhões de pessoas, infelizmente, foram expostas ao frio extremo devido a interrupções de energia ${ }^{47}$. Esse estudo revelou uma anormalidade na formação de impressões digitais das proles de mães expostas entre as semanas $14 \mathrm{e}$ 22 de gravidez. Curiosamente, o período de formação da impressão digital é reconhecidamente o mesmo de formação do hipocampo ${ }^{73,74}$. Sendo assim, é possível que mudanças no hipocampo possam ser encontradas nos mesmos indivíduos. O projeto ainda está em execução e atualmente utiliza abordagem de imagem por ressonância magnética (IRM) para determinar se problemas comportamentais e psicológicos vistos na prole estão ligados a mudanças na morfologia cerebral. Como o hipocampo é responsável pela formação de memória e lógica cognitiva, essa é uma região de interesse primário.

Deve ser reconhecido que os efeitos de um estressor em particular sobre a mãe não depende apenas da natureza do estressor em si, mas também da severidade da ameaça do estressor percebida pela mãe e pela tolerância ao estresse materna e seu comportamento na resposta ao estressor. 


\section{Potenciais vantagens do estresse}

Um interessante e potencialmente útil aspecto da resposta ao estresse é que durante o estresse agudo o giro denteado do hipocampo é mais ativo. Essa é a área do cérebro responsável pelo aprendizado, memória e neurogênese. Em resposta a um estímulo estressor, as células progenitoras neurais no giro denteado são transformadas em neurônios e glia, os quais eventualmente levam a novas conexões sinápticas. Supõe-se que esse seja, ao menos em parte, o mecanismo de como as memórias são feitas, e esse processo capacita indivíduos a lembrarem do objeto ou evento causador do estresse. Uma entrada emocional da amígdala e o reconhecimento do estresse pelo hipocampo cooperam para formar uma memória de medo, e isso esperançosamente levará o indivíduo a evitar uma situação similar no futuro ${ }^{75}$.

Em contraste, o estresse crônico aparentemente diminui a habilidade em formar memórias. Conrad et al. (1996)76 mostraram que ratos cronicamente estressados obtiveram pior performance em teste em labirinto do que aqueles não estressados. Em adição, estudos em humanos mostraram que anos de vida estressante podem levar a um aumento na incidência de depressão e outras condições psiquiátricas ${ }^{77}$. Isso leva à possibilidade de que o estresse agudo possa ser benéfico e o estresse crônico, danoso.

\section{Efeitos do estresse na resistência à insulina e no eixo HPA}

Em humanos, estresse maternal pré-natal durante a gravidez mostrou predispor a baixo peso no nascimento e nascimento prematuro ${ }^{21}$. Estudos mostram que isso, por sua vez, está ligado a disfunções metabólicas, tais como menor tolerância à glicose e resistência à insulina ${ }^{78}$. Usando um teste oral de tolerância à glicose, um estudo recente analisou os níveis de glucose em adultos jovens cujas mães haviam experimentado eventos estressantes durante a gravidez, tais como conflitos na relação, morte ou doença severa de parentes ou amigos próximos, dificuldade financeira severa e acidente de carro, em comparação com indivíduos controle cujas mães tiveram gravidez relativamente livre de estresse ${ }^{79}$. Os resultados mostraram que não havia diferença significativa entre os níveis de glicose dos dois grupos, embora os indivíduos com mães que sofreram estresse pré-natal apresentassem níveis de insulina significativamente mais altos ao ponto de 120 minutos após a administração do teste de tolerância à glicose.

Interessantemente, outro estudo dos mesmos autores mostrou que adultos cujas mães foram estressadas durante a gravidez tinham maiores níveis de cortisol na resposta ao Trier Social Stress Test (TSST), com uma diminuição da resposta ao cortisol após administração de um teste de estimulação com ACTH, indicando uma possível desregulação do eixo HPA ${ }^{80}$. Isso gera evidência, em humanos, de uma associação entre estresse pré-natal e alterações do eixo HPA da prole (Figura 1).

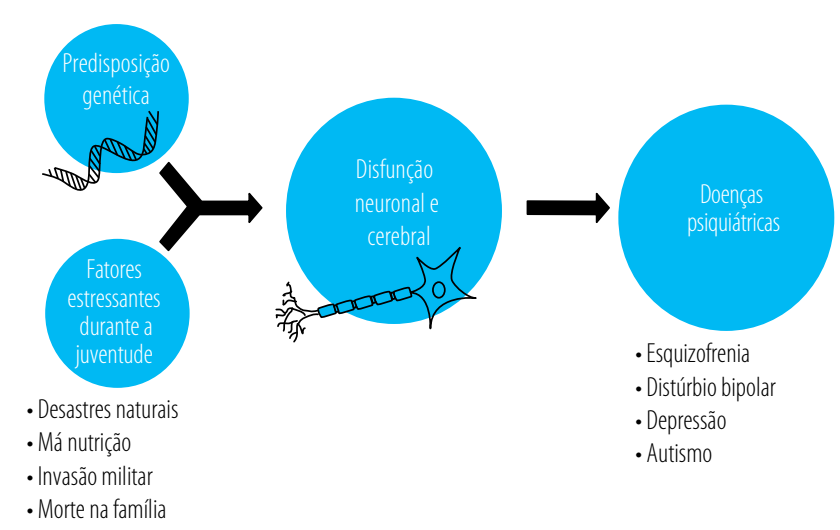

Figura 1. Diagrama esquemático mostrando interações entre predisposição genética e fatores ambientais no início da vida sobre a função neuronal e posterior desenvolvimento de doenças psiquiátricas.
Um estudo usando ratos como modelos mostrou que o estresse pré-natal induz mudanças de longo prazo no comportamento alimentar, metabolismo de glicose e sinalização de insulina ${ }^{81}$. Esses desvios do uso normal da glicose e da insulina são similares àqueles vistos no diabetes tipo II. Os autores especularam que isso está ligado ao aumento dos níveis de glicocorticoides no ambiente intrauterino que acompanha o estresse pré-natal. Outro estudo testou os efeitos da administração de hormônios de estresse diretamente em ovelhas nos estágios iniciais de gravidez, com particular interesse nos efeitos que isso teria na regulação da sinalização de glicose e insulina na prole masculina ${ }^{82}$. O estudo mostrou que a prole masculina de mães nas quais foram administrados hormônios de estresse durante a gravidez apresentava problemas de intolerância à glucose e hiperinsulinemia. Isso sugere que a exposição a glicocorticoide no início da gravidez poderia levar a diabetes e problemas pancreáticos em longo prazo, consistente com o fato de que o estresse durante o começo da gravidez contribui para tais ocorrências em humanos.

\section{Efeitos da sinalização de insulina e o eixo HPA na esquizofrenia}

Apesar de décadas de pesquisa, a fisiopatologia e a etiologia da esquizofrenia e outras desordens psiquiátricas não são completamente entendidas. As principais hipóteses têm se focado nas alterações de sistemas de neurotransmissores tais como as vias glutamatérgica e dopaminérgica, e os atuais medicamentos antipsicóticos têm como principais alvos esses sistemas ${ }^{83}$. Entretanto, esquizofrenia é geralmente associada como manifestações periféricas, incluindo hiperinsulinemia e diabetes melito tipo $\mathrm{II}^{84}$. Embora esses efeitos possam resultar de medicações antipsicóticas, eles foram observados décadas antes do desenvolvimento e uso clínico de antipsicóticos. Em adição, evidência recente de que pacientes podem mostrar esses efeitos ao primeiro sinal clínico e anteriormente ao recebimento do medicamento emergiu ${ }^{85,86}$, e análises sanguíneas demonstraram hiperinsulinemia e anormalidades na secreção de outros fatores endócrinos na primeira apresentação de sintomas ${ }^{87,88}$.

Em um recente estudo de 66 pacientes com primeira manifestação de esquizofrenia em comparação com 68 indivíduos controle, usamos uma série de imunoensaios para medir os níveis de insulina, pró-insulina e des-31,32 pró-insulina empregando fluorescência resolvida no tempo e diferentes combinações de anticorpos monoclonais que discriminassem entre formas específicas da molécula ${ }^{89,87}$, bem como o peptídeo $\mathrm{C}$ e o grânulo secretor de insulina proteína cromogranina A, usando imunoensaios comercialmente disponíveis. Todas essas moléculas foram encontradas em níveis significativamente elevados na circulação de pacientes esquizofrênicos. Em contraste, os níveis de glucose foram relativamente normais, o que foi consistente como a possibilidade de que ao menos alguns desses pacientes fossem insulinorresistentes no início da doença. Isso poderia ter importantes implicações, já que elevados níveis de insulina podem ter efeitos deletérios na função cerebral ${ }^{90}$.

Assumindo que os achados dos níveis aumentados de insulina em pacientes esquizofrênicos estejam associados com um comprometimento na sinalização de insulina, testamos a possibilidade de que a secreção de outros hormônios do sistema neuroendócrino difuso e o eixo HPA estivessem afetados na esquizofrenia. Realizamos uma análise com imunoensaio multiplex de 21 hormônios e moléculas de hormônios relacionadas usando soro sanguíneo de 236 pacientes com primeira manifestação de esquizofrenia em comparação com 230 indivíduos controles usando a plataforma Multi-Analyte Profiling (MAPтM) da Rules Based Medicine (Austin, TX, USA). A análise usando a tecnologia de imunoensaio multiplex revelou que as concentrações séricas de insulina e cromogranina A foram elevadas em indivíduos esquizofrênicos, consistente com os achados citados. Em adição, encontramos concentrações elevadas de polipeptídeo pancreático, prolactina, progesterona e cortisol e níveis diminuídos de hormônio de crescimento (Figura 2) ${ }^{88}$.

Outros pesquisadores encontraram níveis mais altos de $\mathrm{CRH}$, arginina vasopressina (AVP), ACTH e cortisol em estudos de es- 


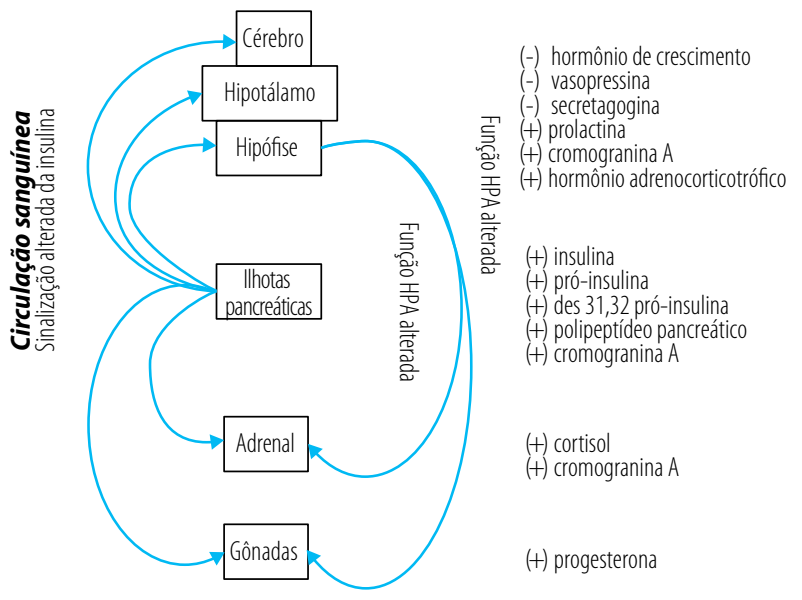

Figura 2. Diagrama esquemático mostrando os potenciais efeitos da resistência à insulina na secreção de outros hormônios e outras moléculas bioativas por meio do sistema neuroendócrino difuso.

quizofrenia e outras condições psiquiátricas ${ }^{91-93}$. Estudos de ressonância magnética funcional (RMf) identificaram volumes pituitários maiores em pacientes com primeira manifestação de esquizofrenia e pituitários menores naqueles indivíduos como manifestação crônica, o que pode indicar uma adaptação, efeitos da medicação ou dessensibilização à hiperatividade do eixo HPA ${ }^{94}$.

Muitos hormônios são afetados pelos ritmos circadiano e ultradiano. Dessa forma, é provável que moléculas identificadas nos estudos acima sejam correguladas em um circuito feedforwardfeedback entre o pâncreas endócrino, pituitária e outros tecidos dos sistemas HPA e hipotálamo-pituitário-gonadal (HPG) ${ }^{95}$. Como um caso no ponto, níveis elevados de insulina têm sido associados com aumentada secreção de prolactina ${ }^{96}$ e diminuídos pulsos de secreção de hormônio de crescimento ${ }^{97}$. Além disso, estudos usando rato com resistência à insulina induzida por dieta como modelo encontraram aumentados níveis de insulina e progesterona ${ }^{98}$. Também, reportamos previamente a corregulação dos níveis de expressão de insulina, hormônio de crescimento, leptina e cortisol em paciente com primeira manifestação de esquizofrenia, usando análise direcionada de conjuntos, a qual detecta padrões de comportamento 99 . O achado de níveis superiores de cortisol usando esse método é consistente com outros estudos ${ }^{100}$.

Também analisamos pituitárias post-mortem de pacientes esquizofrênicos usando uma combinação de cromatografia líquida e espectrometria de massas em tandem, eletroforese diferencial em gel de duas dimensões (2D-DIGE) e imunoensaios multiplex ${ }^{88}$. Usando esses diversos métodos, identificamos mudanças em cortisol, ACTH, AVP, proteína agouti-relacionada, hormônio de crescimento, prolactina e secretagonina. $\mathrm{O}$ achado dos níveis elevados ACTH e cortisol suporta a hipótese de que a hiperatividade do eixo HPA possa estar envolvida na fisiopatologia da doença ${ }^{101}$. Estudos anteriores ligaram níveis anormais de AVP a mudanças de humor e comportamento $^{102}$ e desordens psiquiátricas ${ }^{103,104}$. Em adição, AVP pode afetar a sensibilidade do eixo HPA, já que aparentemente há uma correlação positiva entre os níveis circulantes de AVP, ACTH e cortisol em pacientes esquizofrênicos ${ }^{101}$. Os achados que algumas dessas mudanças também foram encontradas na circulação de indivíduos esquizofrênicos (ver acima) sugerem que eles possam ter um papel na fisiopatologia da doença e poderiam levar à tradução dessas moléculas como biomarcadores periféricos para a esquizofrenia.

\section{Implicações terapêuticas}

A descoberta de que a hiperinsulinemia poderia ter um papel em indivíduos esquizofrênicos de início tardio com mães estressadas no período pré-natal sugere que drogas que melhoram a sinalização da insulina e o uso da glicose podem representar uma nova estratégia de tratamento em potencial. No caso de desordens psiquiátricas como a esquizofrenia, estratégias terapêuticas que possuem como alvo disfunções metabólicas inerentes poderiam se tornar uma alternativa efetiva a medicações antipsicóticas tradicionais ${ }^{87}$. Essa possibilidade é suportada pelo achado de que agentes sensibilizantes a insulina metformina e rosiglitazona podem corrigir a resistência à insulina, que aparece conjuntamente ao tratamento antipsicótico, sem inibir a eficiência da droga antipsicótica no tratamento da doença ${ }^{105}$.

Estratégias semelhantes estão provando ser frutíferas para o tratamento de déficit de memória e mal de Alzheimer. Testes clínicos estão focados no uso de agonistas de receptores ativados por proliferadores peroxissomais gama (PPAR $\gamma$ ) tais como rosiglitazona e pioglitazona como uma terapia alternativa para melhorar a cognição ${ }^{106}$. Agonistas de PPAR $\gamma$ induzem a transcrição de genes específicos, levando ao aumento da sensibilidade à insulina, entre outros efeitos, e foram primeiramente usados em testes clínicos com o objetivo de tratar diabetes melito e aterosclerose (http://www.diabetesselfmanagement. com/Articles/Diabetes-Definitions/ppar_agonists/). Um grupo de pesquisa conduziu um teste controlado, randômico, de seis meses, em pacientes sofrendo de doença de Alzheimer em grau moderado e diabetes tipo $\mathrm{II}^{107}$. Pacientes foram assinalados randomicamente a um de dois grupos. Um dos grupos foi tratado com 15-30 mg de pioglitazona, uma vez por dia, e outro foi utilizado como controle. O grupo que recebeu pioglitazona mostrou melhora na função cognitiva e aumento do fluxo sanguíneo no lobo temporal do cérebro, enquanto o grupo controle não mostrou melhoras. Foi interessante que os efeitos da pioglitazona foram acompanhados pelo aumento do fluxo sanguíneo, já que isso poderia potencialmente levar a um aumento da quantidade de glicose disponível para o cérebro.

O esteroide adrenal de-hidroepiandrosterona (DHEA) possui propriedades antiglicocorticoides que provam ser úteis na regulação de altos níveis de cortisol e ação de glicocorticoides nos cérebros de pacientes psiquiátricos ${ }^{108}$. Estudos usando DHEA em adição à medicação antipsicótica em pacientes esquizofrênicos já sob tratamento medicamentoso encontraram uma melhora significativa nos sintomas negativos, depressivos e de ansiedade da doença ${ }^{109}$. O tratamento com DHEA também produziu melhora nos efeitos colaterais extrapiramidais, tais como tremores involuntários associados com o tratamento antipsicótico ${ }^{110}$.

\section{Conclusões e perspectivas futuras}

O estresse pré-natal tem sido ligado a muitos problemas comportamentais e psicológicos, tais como esquizofrenia, TDAH, autismo e depressão. Mudanças são vistas nos cérebros de uma variedade de modelos animais em resposta ao estresse pré-natal. Em particular, mudanças são vistas no hipocampo, amígdala, corpo caloso, córtex cerebral, cerebelo e hipotálamo. Essas áreas do cérebro são responsáveis pelo controle do comportamento, e suas alterações poderiam explicar os problemas psicológicos testemunhados, embora isso não tenha sido provado ocorrer em humanos. Os mecanismos pelos quais essas mudanças são alcançadas no cérebro muito provavelmente envolvem os eixos HPA materno e fetal e o efeito do ambiente intrauterino no desenvolvimento do cérebro fetal. Independentemente do efeito do ambiente no desenvolvimento do feto, é provável que haja também um elemento genético para contrair tais problemas.

Aparentemente, o tempo de ocorrência e a gravidade do estresse experimentado pelas mães têm impacto no desenvolvimento de problemas comportamentais e psicológicos por parte do feto. Entretanto, posterior pesquisa é necessária antes que os exatos efeitos do tempo de ocorrência e gravidade possam ser determinados. Há uma quantidade cada vez maior de evidência de componentes metabólicos e hormonais em condições como a esquizofrenia, os quais em alguns casos podem estar associados ao estresse pré-natal. Anormalidades no metabolismo de glicose e sinalização de insulina e no eixo HPA parecem estar presentes nos estados iniciais dessas doenças e podem prover a base para o desenvolvimento de biomarcadores, muito necessitados nas condições psiquiátricas. $\mathrm{O}$ uso de tais biomarcadores 
poderia levar a um melhor diagnóstico e à orientação de pacientes a estratégias medicinais de maneira personalizada (a droga certa para o paciente certo no tempo certo), bem como à possibilidade de tratamento preventivo. Dado o potencial dessa linha de pesquisa para melhorar o diagnóstico e criar estratégias de tratamento alternativas, pesquisas futuras devem ser feitas nesta direção.

É claro, entretanto, que o gatilho ambiental do estresse intrauterino não é o único catalisador para o desenvolvimento da doença. É provável que haja também uma predisposição genética, que, quando combinada com o gatilho ambiental, leva ao desenvolvimento da doença. Isso está em acordo com a hipótese de duas causas. Temos a tendência de desconsiderar nossas respostas emocionais como tristeza ou estresse como se elas não fossem tangíveis. Entretanto, tudo que sentimos é devido ao movimento de substâncias químicas dentro de nós. Essas substâncias, neurotransmissores e hormônios, produzem a sensação de estresse, mas também levam a uma cascata de outras reações no corpo, as quais podem ter consequências muito reais e tangíveis.

\section{Agradecimentos}

Esta pesquisa foi suportada pelo Instituto de Pesquisa Médica Stanley (SMRI) e pelo programa de pesquisa da União Europeia FP7 SchizDX (referência do projeto 223427).

\section{Referências}

1. Barker DJ, Winter PD, Osmond C, Margetts B, Simmonds SJ. Weight in infancy and death from ischaemic heart disease. Lancet. 1989;2:577-80.

2. Hales CN, Barker DJ. Type 2 (non-insulin-dependent) diabetes mellitus: the thrifty phenotype hypothesis. Diabetologia. 1992;35:595-601.

3. Owen MJ, O'Donovan MC, Thapar A, Craddock N. Neurodevelopmental hypothesis of schizophrenia. Br J Psychiatry. 2011;198:173-5.

4. Barker DJ. Maternal nutrition, fetal nutrition, and disease in later life. Nutrition. 1997;13:807.

5. Lovejoy DA. Neuroendocrinology, an integrated approach. Chichester: John Wiley \& Sons Ltd.; 2005. p. 243-56.

6. Perry BD, Pollard R. Homeostasis, stress, trauma, and adaptation. A neurodevelopmental view of childhood trauma. Child Adolesc Psychiatr Clin N Am. 1998;7:33-51, viii.

7. Vitaliano PP, Maiuro RD, Mitchell E, Russo J. Perceived stress in medical school: resistors, persistors, adaptors and maladaptors. Soc Sci Med. 1989;28:1321-9.

8. Vaz RF, Mbajiorgu EF, Acuda SW. A preliminary study of stress levels among first year medical students at the University of Zimbabwe. Cent Afr J Med. 1998;44:214-9.

9. Stuber ML. Psychiatric sequelae in seriously ill children and their families. Psychiatr Clin North Am. 1996;19:481-93.

10. Simmons BL, Nelson DL. Eustress at work: the relationship between hope and health in hospital nurses. Health Care Manage Rev. 2001;26:7-18.

11. Seyle H. Stress and distress. Compr Ther. 1975;1:9-13.

12. Arun CP. Fight or flight, forbearance and fortitude: the spectrum of actions of the catecholamines and their cousins. Ann N Y Acad Sci. 2004;1018:137-40.

13. Stratakis CA, Chrousos GP. Neuroendocrinology and pathophysiology of stress. Ann NY Acad Sci. 1995;771:1-18.

14. Vaccaro KK, Liang BT, Sheard BE, Perlman RL. Monensin inhibits catecholamine synthesis in pheochromocytoma cells. J Pharmacol Exp Ther. 1982;221:536-40.

15. Halter JB, Beard JC, Porte D Jr. Islet function and stress hyperglycemia: plasma glucose and epinephrine interaction. Am J Physiol. 1984;247(Part 1):E47-52.

16. Charil A, Laplante DP, Vaillancourt C, King S. Prenatal stress and brain development. Brain Res Rev. 2010;65:56-79.

17. Ng PC. Effect of stress on the hypothalamic-pituitary-adrenal axis in the fetus and newborn. J Pediatr. 2011;158(2 Suppl):e41-3.

18. Seckl JR, Holmes MC. Mechanisms of disease: glucocorticoids, their placental metabolism and fetal 'programming' of adult pathophysiology. Nat Clin Pract Endocrinol Metab. 2007;3:479-88.
19. Wadhwa PD, Porto M, Chicz-DeMet A, Sandman CA. Maternal CRH levels in early third trimester predict length of gestation in human pregnancy. Am J Obstet Gynecol. 1998;179:1079-85.

20. Kastin AJ, Akerstrom V. Differential interactions of urocortin/corticotropin-releasing hormone peptides with the blood-brain barrier. Neuroendocrinology. 2002;75:367-74.

21. Wadhwa PD, Sandman CA, Garite TJ. The neurobiology of stress in human pregnancy: implications for prematurity and development of the fetal central nervous system. Prog Brain Res. 2001;133:131-42.

22. Sandman CA, Wadhwa PD, Glynn L, Chicz-Demet A, Porto M, Garite TJ. Corticotrophin-releasing hormone and fetal responses in human pregnancy. Ann NY Acad Sci. 1999;897:66-75.

23. Schneider ML. Delayed object permanence development in prenatally stressed rhesus monkey infants (Macaca mulatta). Occup Ther J Res. 1992;12:96-110.

24. Schneider ML, Coe CL. Repeated social stress during pregnancy impairs neuromotor development in the primate infant. J Dev Behav Pediatr. 1993;14:81-7.

25. Clarke AS, Schneider ML. Prenatal stress has long-term effects on behavioral responses to stress in juvenile rhesus monkeys. Dev Psychobiol. 1993;26:293-304.

26. Clarke AS, Wittwer DJ, Abbott DH, Schneider ML. Long-term effects of prenatal stress on HPA axis activity in juvenile rhesus monkeys. Dev Psychobiol. 1994;27:257-69.

27. Li J, Olsen J, Vestergaard M, Obel C. Attention-deficit/hyperactivity disorder in the offspring following prenatal maternal bereavement: a nationwide follow-up study in Denmark. Eur Child Adolesc Psychiatry. 2010;19:747-53.

28. Grizenko N, Shayan YR, Polotskaia A, Ter-Stepanian M, Joober R. Relation of maternal stress during pregnancy to symptom severity and response to treatment in children with ADHD. J Psychiatry Neurosci. 2008;33:10-6.

29. Linnet KM, Dalsgaard S, Obel C, Wisborg K, Henriksen TB, Rodriguez A, et al. Maternal lifestyle factors in pregnancy risk of attention deficit hyperactivity disorder and associated behaviors: review of the current evidence. Am J Psychiatry. 2003;160:1028-40.

30. Van Os J, Selten JP. Prenatal exposure to maternal stress and subsequent schizophrenia. The invasion of The Netherlands. Br J Psychiatry. 1998;172:324-6.

31. Susser E, Neugebauer R, Hoek HW, Brown AS, Lin S, Labovitz D, et al. Schizophrenia after prenatal famine. Further evidence. Arch Gen Psychiatry. 1996;53:25-31.

32. Brown AS, Susser ES. Prenatal nutritional deficiency and risk of adult schizophrenia. Schizophr Bull. 2008;34:1054-63.

33. Hoek HW, Brown AS, Susser. The Dutch famine and schizophrenia spectrum disorders. Soc Psychiatry Psychiatr Epidemiol. 1998;33:373-9.

34. Kahn HS, Graff M, Stein AD, Lumey LH. A fingerprint marker from early gestation associated with diabetes in middle age: the Dutch Hunger Winter Families Study. Int J Epidemiol. 2009;38:101-9.

35. St Clair D, Xu M, Wang P, Yu Y, Fang Y, Zhang F, et al. Rates of adult schizophrenia following prenatal exposure to the Chinese famine of 1959-1961. JAMA. 2005;294:557-62.

36. Huttenen MO, Niskanen P. Prenatal loss of father and psychiatric disorders. Arch Gen Psychiatry. 1978;35:429-31.

37. Kinney DK, Hyman W, Greetham C, Tramer S. Increased relative risk for schizophrenia and prenatal exposure to a severe tornado. Schizophr Res. 1999;36:45.

38. Cripps RL, Martin-Gronert MS, Archer ZA, Hales CN, Mercer JG, Ozanne SE. Programming of hypothalamic neuropeptide gene expression in rats by maternal dietary protein content during pregnancy and lactation. Clin Sci (Lond). 2009;117:85-93.

39. Torres N, Bautista CJ, Tovar AR, Ordáz G, Rodríguez-Cruz M, Ortiz V, et al. Protein restriction during pregnancy affects maternal liver lipid metabolism and fetal brain lipid composition in the rat. Am J Physiol Endocrinol Metab. 2010;298:E270-7.

40. Palmer AA, Printz DJ, Butler PD, Dulawa SC, Printz MP. Prenatal protein deprivation in rats induces changes in prepulse inhibition and NMDA receptor binding. Brain Res. 2004;996:193-201.

41. Palmer AA, Brown AS, Keegan D, Siska LD, Susser E, Rotrosen J, et al. Prenatal protein deprivation alters dopamine-mediated behaviors and dopaminergic and glutamatergic receptor binding. Brain Res. 2008;1237:62-74. 
42. Csomor PA, Yee BK, Feldon J, Theodoridou A, Studerus E, Vollenweider VX. Impaired prepulse inhibition and prepulse-elicited reactivity but intact reflex circuit excitability in unmedicated schizophrenia patients: a comparison with healthy subjects and medicated schizophrenia patients. Schizophr Bull. 2009;35:244-55.

43. Watson JB, Mednick SA, Huttunen M, Wang X. Prenatal teratogens and the development of adult mental illness. Dev Psychopathol. 1999;11:457-66.

44. Weinstock M. The long-term behavioural consequences of prenatal stress. Neurosci Biobehav Rev. 2008;32:1073-86.

45. Kinney DK, Miller AM, Crowley DJ, Huang E, Gerber E. Autism prevalence following prenatal exposure to hurricanes and tropical storms in Louisiana. J Autism Dev Disord. 2008;38:481-8.

46. Beversdorf DQ, Manning SE, Hillier A, Anderson SL, Nordgren RE, Walters SE, et al. Timing of prenatal stressors and autism. J Autism Dev Disord. 2005;35:471-8.

47. King S, Laplante DP. The effects of prenatal maternal stress on children's cognitive development: Project Ice Storm. Stress. 2005;8:35-45.

48. King S, Mancini-Marie A, Brunet A, Walker E, Meaney MJ, Laplante DP. Prenatal maternal stress from a natural disaster predicts dermatoglyphic asymmetry in humans. Dev Psychopathol. 2009;21:343-53.

49. Laplante DP, Barr RG, Brunet A, Galbaud du Fort G, Meaney ML, Saucier $\mathrm{JF}$, et al. Stress during pregnancy affects general intellectual and language functioning in human toddlers. Pediatr Res. 2004;56:400-10.

50. Laplante DP, Brunet A, Schmitz N, Ciampi A, King S. Project Ice Storm: prenatal maternal stress affects cognitive and linguistic functioning in 5 1/2-year-old children. J Am Acad Child Adolesc Psychiatry. 2008;47:1063-72.

51. Talge NM, Neal C, Glover V. Antenatal maternal stress and long-term effects on child neurodevelopment: how and why? J Child Psychol Psychiatry. 2007;48:245-61.

52. Deminière JM, Piazza PV, Guegan G, Abrous N, Maccari S, Le Moal M, et al. Increased locomotor response to novelty and propensity to intravenous amphetamine self-administration in adult offspring of stressed mothers. Brain Res. 1992;586:135-9.

53. Uno H, Tarara R, Else J, Sulemen M, Sapolsky RM. Hippocampal damage associated with prolonged and fatal stress in primates. J Neurosci. 1989;9:1705-11.

54. Uno H, Lohmiller L, Thieme C, Kemnitz JW, Engle MJ, Roecker EB, et al. Brain damage induced by prenatal exposure to dexamethasone in fetal rhesus macaques, 1. Hippocampus Dev Brain Res. 1990;53:157-67.

55. Uno H, Eisele S, Sakai A, Shelton S, Baker E, DeJesus O, et al. Neurotoxicity of glucocorticoids in the primate brain. Horm Behav. 1994;28:336-48.

56. Schmitz C, Rhodes ME, Bludau M, Kaplan S, Ong P, Ueffing I, et al. Depression: reduced number of granule cells in the hippocampus of female, but not male, rats due to prenatal restraint stress. Mol Psychiatry. 2002;7:810-3.

57. Coe CL, Kramer M, Czéh B, Gould E, Reeves AJ, Kirschbaum C, et al. Prenatal stress diminishes neurogenesis in the dentate gyrus of juvenile rhesus monkeys. Biol Psychiatry. 2003;54:1025-34.

58. Salm AK, Pavelko M, Krouse EM, Webster W, Kraszpulski M, Birkle DL. Lateral amygdaloid nucleus expansion in adult rats is associated with exposure to prenatal stress. Brain Res Dev Brain Res. 2004;148:159-67.

59. Kraszpulski M, Dickerson PA, Salm AK. Prenatal stress affects the developmental trajectory of the rat amygdala. Stress. 2006;9:85-95.

60. Kawamura T, Chen J, Takahashi T, Ichitani Y, Nakahara D. Prenatal stress suppresses cell proliferation in the early developing brain. Neuroreport. 2006;17:1515-8.

61. Coe CL, Lulbach GR, Schneider ML. Prenatal disturbance alters the size of the corpus callosum in young monkeys. Dev Psychobiol. 2002;41:178-85.

62. Poland RE, Cloak C, Lutchmansingh PJ, McCracken JT, Chang L, Ernst T. Brain N-acetyl aspartate concentrations measured by H MRS are reduced in adult male rats subjected to perinatal stress: preliminary observations and hypothetical implications for neurodevelopmental disorders. J Psychiatr Res. 1999;33:41-51.

63. Barros VG, Duhalde-Vega M, Caltana L, Brusco A, Antonelli MC. Astrocyte-neuron vulnerability to prenatal stress in the adult rat brain. J Neurosci Res. 2006;83:787-800.

64. Ulupinar E, Yucel F. Prenatal stress reduces interneuronal connectivity in the rat cerebellar granular layer. Neurotoxicol Teratol. 2005;27:475-84.

65. Ulupinar E, Yucel F, Ortug G. The effects of prenatal stress on the Purkinje cell neurogenesis. Neurotoxicol Teratol. 2006;28:86-94.
66. Anderson DK, Rhees RW, Fleming DE. Effects of prenatal stress on differentiation of the sexually dimorphic nucleus of the preoptic area (SDN-POA) of the rat brain. Brain Res. 1995;332:113-8.

67. Kerchner M, Ward IL. SDN-MPOA volume in male rats is decreased by prenatal stress, but is not related to ejaculatory behavior. Brain Res. 1992;581:244-51.

68. Egaas B, Courchesne E, Saitoh O. Reduced size of corpus callosum in autism. Arch Neurol. 1995;52:794-801.

69. Seidman LJ, Valera EM, Makris N. Structural brain imaging of attentiondeficit/hyperactivity disorder. Biol Psychiatry. 2005;57:1263-72.

70. Innocenti GM, Ansermet F, Parnas J. Schizophrenia, neurodevelopment and corpus callosum. Mol Psychiatry. 2003;8:261-74.

71. Lemaire V, Koehl M, Le Moal M, Abrous DN. Prenatal stress produces learning deficits associated with an inhibition of neurogenesis in the hippocampus. Proc Natl Acad Sci U S A. 2000;97:11032-7.

72. Ward HE, Johnson EA, Salm AK, Birkle DL. Effects of prenatal stress on defensive withdrawal behavior and corticotropin releasing factor systems in rat brain. Physiol Behav. 2000;70:359-66.

73. Bayer SA, Altman J, Russo RJ, Zhang X. Timetables of neurogenesis in the human brain based on experimentally determined patterns in the rat. Neurotoxicology. 1993;14:83-144.

74. Van Oel CJ, Baare WF, Hulshoff Pol HE, Haag J, Balazs J, Dingemans A, et al. Differentiating between low and high susceptibility to schizophrenia in twins: the significance of dermatoglyphic indices in relation to other determinants of brain development. Schizophr Res. 2001;52:181-93.

75. Reul JM, Chandramohan Y. Epigenetic mechanisms in stress-related memory formation. Psychoneuroendocrinology. 2007;32(Suppl 1):S21-5.

76. Conrad CD, Galea LAM, Kuroda Y, McEwen BS. Chronic stress impairs rat spatial memory on the $\mathrm{Y}$ maze, and this effect is blocked by tianeptine treatment. Behav Neurosci. 1996;110:1321-34.

77. Marin MF, Lord C, Andrews J, Juster RP, Sindi S, Arsenault-Lapierre G, et al. Chronic stress, cognitive functioning and mental health. Neurobiol Learn Mem. 2011;96(4):583-95.

78. Newsome CA, Shiell AW, Fall CH, Phillips DI, Shier R, Law CM. Is birth weight related to later glucose and insulin metabolism? A systematic review. Diabet Med. 2003;20:339-48.

79. Entringer S, Wüst S, Kumsta R, Layes IM, Nelson EL, Hellhammer DH, et al. Prenatal psychosocial stress exposure is associated with insulin resistance in young adults. Am J Obstet Gynecol. 2008;199:498.e1-7

80. Entringer S, Kumsta R, Hellhammer DH, Wadhwa PD, Wüst S. Prenatal exposure to maternal psychosocial stress and HPA axis regulation in young adults. Horm Behav. 2009;55:292-8.

81. Lesage J, Del-Favero F, Leonhardt M, Louvart H, Maccari S, Vieau D, et al. Prenatal stress induces intrauterine growth restriction and programmes glucose intolerance and feeding behaviour disturbances in the aged rat. J Endocrinol. 2004;181:291-6.

82. De Blasio MJ, Dodic M, Jefferies AJ, Moritz KM, Wintour EM, Owens JA. Maternal exposure to dexamethasone or cortisol in early pregnancy differentially alters insulin secretion and glucose homeostasis in adult male sheep offspring. Am J Physiol Endocrinol Metab. 2007;293:E75-82.

83. Biedermann F, Fleischhacker WW. Antipsychotics in the early stage of development. Curr Opin Psychiatry. 2009;22:326-30.

84. Hasnain M, Fredrickson SK, Vieweg WV, Pandurangi AK. Metabolic syndrome associated with schizophrenia and atypical antipsychotics. Curr Diab Rep. 2010;10:209-16.

85. Ryan MC, Collins P, Thakore JH. Impaired fasting glucose tolerance in first-episode, drug-naive patients with schizophrenia. Am J Psychiatry. 2003;160:284-9.

86. Spelman LM, Walsh PI, Sharifi N, Collins P, Thakore JH. Impaired glucose tolerance in first-episode drug-naive patients with schizophrenia. Diabet Med. 2007;24:481-5.

87. Guest PC, Wang L, Harris LW, Burling K, Levin Y, Ernst A, et al. Increased levels of circulating insulin-related peptides in first-onset, antipsychotic naïve schizophrenia patients. Mol Psychiatry. 2010;15:118-9.

88. Guest PC, Schwarz E, Krishnamurthy D, Harris LW, Leweke FM, Rothermundt M, et al. Altered levels of circulating insulin and other neuroendocrine hormones associated with the onset of schizophrenia. Psychoneuroendocrinology. 2011;36:1092-6.

89. Sobey WJ, Beer SF, Carrington CA, Clark PM, Frank BH, Gray IP, et al. Sensitive and specific two-site immunoradiometric assays for human insulin, proinsulin, 65-66 split and 32-33 split proinsulins. Biochem J. 1989;260:535-41. 
90. Taguchi A, Wartschow LM, White MF. Brain IRS2 signaling coordinates life span and nutrient homeostasis. Science. 2007;317:369-72.

91. Banki CM, Bissette G, Arato M, O’Connor L, Nemeroff CB. CSF corticotropin-releasing factor-like immunoreactivity in depression and schizophrenia. Am J Psychiatry. 1987;144:873-7.

92. Brunelin J, D’Amato T, Van Os J, Cochet A, Suaud-Chagny MF, Saoud $M$. Effects of acute metabolic stress on the dopaminergic and pituitaryadrenal axis activity in patients with schizophrenia, their unaffected siblings and controls. Schizophr Res. 2008;100:206-11.

93. Tsigos C, Chrousos GP. Hypothalamic-pituitary-adrenal axis, neuroendocrine factors and stress. J Psychosom Res. 2002;53;865-71.

94. Pariante CM, Vassilopoulou K, Velakoulis D, Phillips L, Soulsby B, Wood SJ, et al. Pituitary volume in psychosis. Br J Psychiatry. 2004;185:5-10.

95. Walker JJ, Terry JR, Lightman SL. Origin of ultradian pulsatility in the hypothalamic-pituitary-adrenal axis. Proc Biol Sci. 2010;277:1627-33.

96. Ben-Jonathan N, Hugo ER, Brandebourg TD, LaPensee CR. Focus on prolactin as a metabolic hormone. Trends Endocrinol Metab. 2006;17:110-6.

97. Tannenbaum GS, Martin JB, Colle E. Ultradian growth hormone rhythm in the rat: effects of feeding, hyperglycemia, and insulin-induced hypoglycemia. Endocrinology. 1976;99:720-7.

98. Akamine EH, Marcal AC, Camporez JP, Hoshida MS, Caperuto LC, Bevilacqua E, et al. Obesity induced by high-fat diet promotes insulin resistance in the ovary. J Endocrinol. 2010;206:65-74.

99. Cheng TM, Lu YE, Guest PC, Rahmoune H, Harris LW, Wang L, et al. Identification of targeted analyte clusters for studies of schizophrenia. Mol Cell Proteomics. 2010;9:510-22.

100. Meltzer HY, Lee MA, Jayathilake K. The blunted plasma cortisol response to apomorphine and its relationship to treatment response in patients with schizophrenia. Neuropsychopharmacology. 2001;24:278-90.

101. Ryan MC, Sharifi N, Condren R, Thakore JH. Evidence of basal pituitaryadrenal overactivity in first episode, drug naive patients with schizophrenia. Psychoneuroendocrinology. 2004;29:1065-70.
102. Heinrichs M, Von Dawans B, Domes G. Oxytocin, vasopressin, and human social behavior. Front Neuroendocrinol. 2009;30:548-57.

103. Goldman MB, Robertson GL, Luchins DJ, Hedeker D. The influence of polydipsia on water excretion in hyponatremic, polydipsic, schizophrenic patients. J Clin Endocrinol Metab. 1996;81:1465-70.

104. Mai JK, Berger K, Sofroniew MV. Morphometric evaluation of neurophysin-immunoreactivity in the human brain: pronounced inter-individual variability and evidence for altered staining patterns in schizophrenia. J Hirnforsch. 1993;34:133-54.

105. Bahtiyar G, Weiss K, Sacerdote AS. Novel endocrine disrupter effects of classic and atypical antipsychotic agents and divalproex: induction of adrenal hyperandrogenism, reversible with metformin or rosiglitazone. Endocr Pract. 2007;13:601-8.

106. Sato T, Hanyu H, Hirao K, Kanetaka H, Sakurai H, Iwamoto T. Efficacy of PPAR-gamma agonist pioglitazone in mild Alzheimer disease. Neurobiol Aging. 2011;32(9):1626-33.

107. Landreth G, Jiang QG, Mandrekar S, Heneka M. PPAR gamma agonists as therapeutics for the treatment of Alzheimer's disease. Neurotherapeutics. 2008;5:481-9.

108. Strous RD, Maayan R, Lapidus R, Stryjer R, Lustig M, Kotler M, et al. Dehydroepiandrosterone augmentation in the management of negative, depressive, and anxiety symptoms in schizophrenia. Arch Gen Psychiatry. 2003;60:133-41.

109. Nachshoni T, Ebert T, Abramovitch Y, Assael-Amir M, Kotler M, Maayan R, et al. Improvement of extrapyramidal symptoms following dehydroepiandrosterone (DHEA) administration in antipsychotic treated schizophrenia patients: a randomized, double-blind placebo controlled trial, Schizophr Res. 2005;79:251-6.

110. Hunt PJ, Gurnell EM, Huppert FA, Richards C, Prevost AT, Wass JA, et al. Improvement in mood and fatigue after dehydroepiandrosterone replacement in Addison's disease in a randomized, double blind trial. J Clin Endocrinol. 2000;85:4650-6. 